\title{
The infants who have mature hip on ultrasonography but have risk factors of developmental dysplasia of the hip are required radiographic examination
}

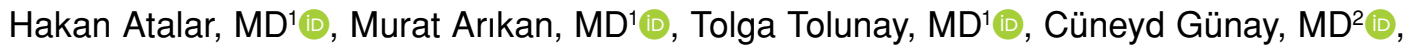 \\ Selçuk Bölükbaşı, MD'두 \\ ${ }^{1}$ Department of Orthopedics and Traumatology, Gazi University Faculty of Medicine, Ankara, Turkey \\ ${ }^{2}$ Department of Orthopedics and Traumatology, Eskişehir Osmangazi University Faculty of Medicine, Eskişehir, Turkey
}

Developmental dysplasia of the hip (DDH) is a devastating disease and early diagnosis is important for the prevention of complications due to $\mathrm{DDH}^{[1,2]}$ Currently, hip ultrasonography by the Graf method is widely used for early diagnosis. Graf et al. ${ }^{[1]}$ described hip ultrasonography method for both early diagnosis and also for the follow-up of treatment of $\mathrm{DDH}^{\left[{ }^{[2]}\right.}$ In this method, the process is carried out on lateral decubitus position and coronal images are taken. Then, three lines are drawn and two angles are measured on sonographic images. The lines are formed as follows: a vertical line is drawn parallel to the lateral border of the ossified ilium, a second line is drawn along the axis of the cartilaginous roof of the acetabulum which extends from bony edge of the acetabulum to the labrum, and a third line is

Received: September 12, 2021

Accepted: October 15, 2020

Published online: November 19, 2021

Correspondence: Cüneyd Günay, MD. Eskișehir Osmangazi Üniversitesi Tıp Fakültesi Ortopedi ve Travmatoloji Anabilim Dall, 26040 Meşelik, Eskişehir, Türkiye.

E-mail: cungunay@hotmail.com

Doi: $10.52312 /$ jdrs.2021.413

Citation: Atalar H, Arıkan M, Tolunay T, Günay C, Bölükbașı S. The infants who have mature hip on ultrasonography but have risk factors of developmental dysplasia of the hip are required radiographic examination. Jt Dis Relat Surg 2021;32(3):598-604.

(02021 All right reserved by the Turkish Joint Diseases Foundation

This is an open access article under the terms of the Creative Commons Attribution-NonCommercial License, which permits use, distribution and reproduction in any medium, provided the original work is properly cited and is not used for commercial purposes (http://creativecommons.org/licenses/by-nc/4.0/).

\section{ABSTRACT}

Objectives: This study aims to evaluate the short-term results of infants who were radiologically diagnosed with developmental dysplasia of the hip (DDH), but in whom hip development was normal ultrasonographically.

Patients and methods: Between January 2018 and September 2020, a total of 15 infants (2 males, 13 females; median age: 5 months; range, 4 to 6 months) who were diagnosed with DDH radiologically and treated were retrospectively analyzed. Hip ultrasonography was used for early diagnosis, treatment, and for follow-up in infants up to six months of age. While the ultrasonographic findings were normal, radiography was performed in infants between four to six months of age who were at risk for DDH.

Results: Fifteen patients (22 hips) were diagnosed with DDH radiologically and treated. Radiologic dysplasia continued in seven hips of five patients during short-term follow-up.

Conclusion: These results suggest that ultrasonographic hip maturation may not be consistent with normal hip development in infants, particularly in those who are at risk for DDH. In infants with DDH which is confirmed by radiography (less than 6 months of age), the diagnosis may be missed on ultrasonographic examination.

Keywords: Congenital hip dysplasia, diagnostic ultrasound, hip joint, radiography.

drawn from the inferior edge of the ilium to the lateral bony edge of the ilium. Then, two angles, which are called as alpha and beta angles, are determined and measured. Alpha angle is formed between the first and third lines, while beta angle is formed between the first and the second lines. ${ }^{[3,4]}$

An alpha angle above 60 degrees ultrasonographically indicates normal hip maturation (Graf type I). The bony rim is angular in type I hips 


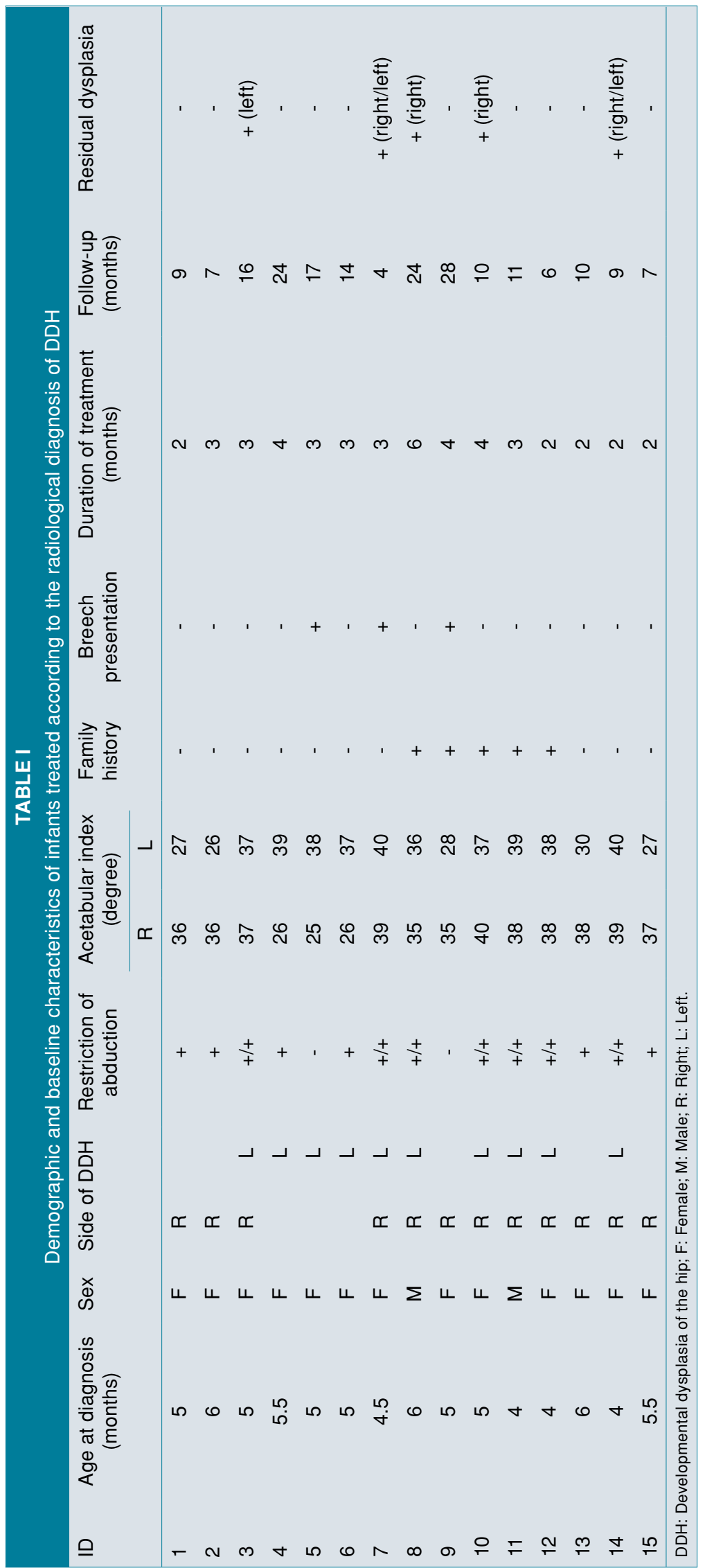


and the bony socket also is well developed. Dr. Graf $^{[3]}$ realized that USG was more useful for the diagnosis of DDH than radiography owing to its capability to visualize soft tissue of the hip joint. Therefore, ultrasonography is used as a screening method for DDH worldwide. In Sweden, Germany and Austria, after a universal screening program, the rate of the incidence of late-diagnosed $\mathrm{DDH}$ cases has dramatically decreased..$^{[5-7]}$ According to the Graf method, a mature hip does not expected to deteriorate over time, unless exceptional situations such as neuromuscular disorders, septic arthritis, and initial misdiagnosis. ${ }^{[3]}$ However, there are some reports in the literature showing that late dysplasia may develop after normal neonatal screening. ${ }^{[8-13]}$ According to Graf, ${ }^{[1]}$ this could only happen due to inappropriate performing of his method.

Recently, it has been shown that some hips treated for DDH remained dysplastic, despite of ultrasonographic maturation. The hips may remain dysplastic, even if the Graf method is applied correctly. ${ }^{[14]}$ In the present study, we aimed to evaluate the short-term results of infants who were radiologically diagnosed with DDH but in whom hip development was normal ultrasonographically.

\section{PATIENTS AND METHODS}

This single-center, retrospective study was conducted at Gazi University Faculty of Medicine, Department of Orthopedics and Traumatology between January 2018 and September 2020. In our center, hip ultrasonography was only used for early diagnosis and follow-up of DDH of infants up to six months of age. Over the last three years in our daily practice, if there is a risk factor such as family history of first-degree relatives (parents, siblings, grandparents, uncles, aunts and their children), breech presentation, and limitation of abduction (less than 60 degrees when tested at 90 degrees of hip flexion) of the hip in terms of DDH in infants older than four months, even if hip ultrasonography is normal, we obtain pelvis radiography routinely. In this study, a total of 41 infants with normal hip ultrasonography who had pelvis anteroposterior radiographs were screened. Of these, 15 ( 2 males, 13 females; median age: 5 months; range, 4 to 6 months) who were diagnosed with DDH radiologically and treated included. A written informed consent was obtained from each parent and/or legal guardian of the patient. The study protocol was approved by the University of Health Sciences, Dr. Abdurrahman Yurtaslan Ankara Oncology Training and Research Hospital Ethics Committee (Date/No: 2020-11/869). The study was conducted in accordance with the principles of the Declaration of Helsinki.

Ultrasonography was performed in all infants with a $7.5-\mathrm{MHz}$ linear transducer (Logiq ${ }^{\mathrm{TM}}$ E; GE Healthcare Co., Ltd, China). All ultrasonography examinations were performed by a single operator using the Graf method. An alpha angle above 60 degrees ultrasonographically was accepted as the mature hip. Anteroposterior radiography of the pelvis was obtained in the supine position. Before evaluation of the radiographs, their compliance with the Tönnis criteria was confirmed. ${ }^{[15]}$ The Hilgenreiner's line was drawn and acetabular index was measured. Lateral acetabular

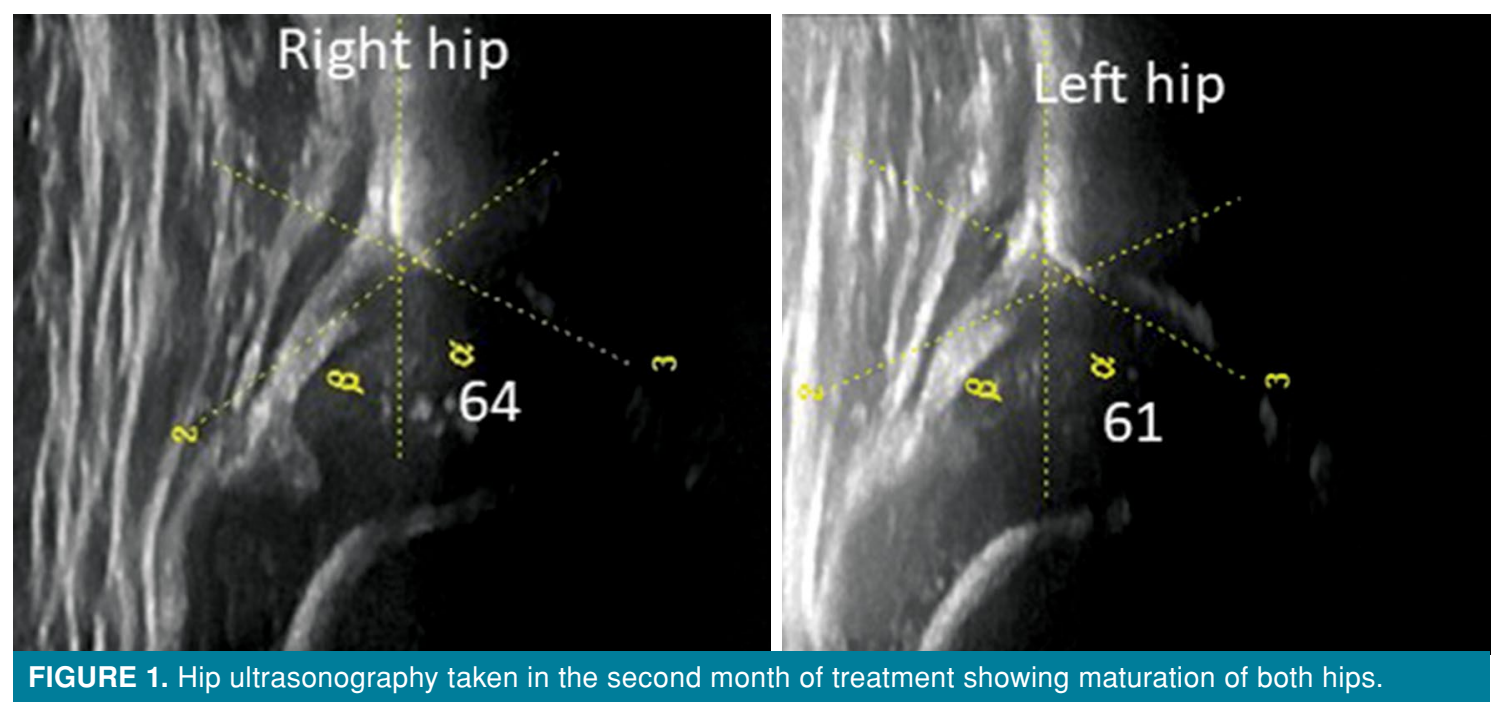




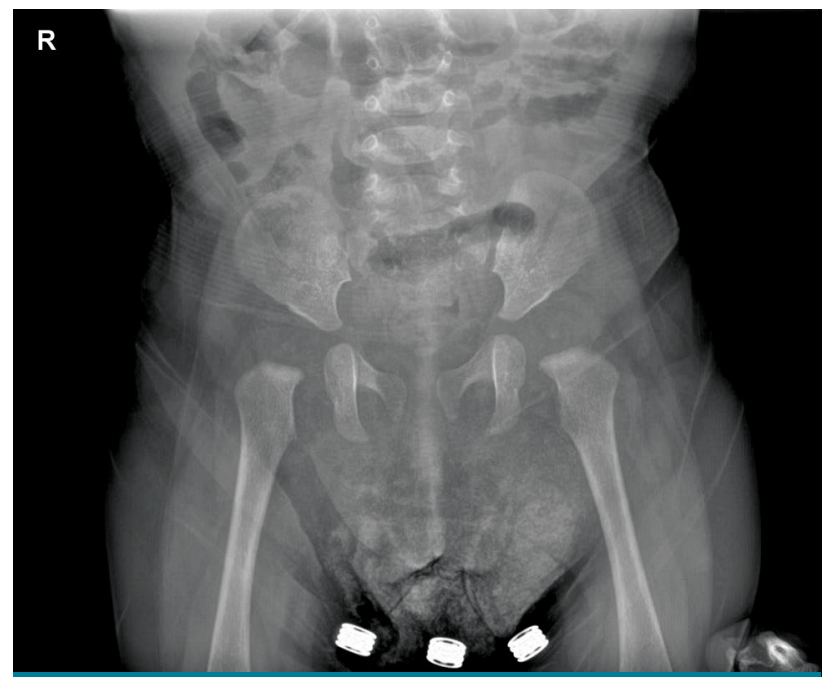

FIGURE 2. Pelvic radiography taken in the second month of treatment showing developmental dysplasia of the hip in both hips.

rim with bony defect, flattened acetabular rim and high acetabular index values according to age (above double standard deviation/according to Tönnis criteria) were accepted as acetabular dysplasia. ${ }^{[15]}$

\section{RESULTS}

Developmental dysplasia of the hip was detected in 22 hips of 15 patients radiologically. These patients were treated conservatively ( 6 patients with abduction orthosis, 7 patients with Tübingen hip flexion splint, and 2 patients with Frejka pillow) and were followed for an median of 12.9 (range, 4 to 28) months. Radiological dysplasia continued in seven hips of five patients in the short-term follow-up, despite

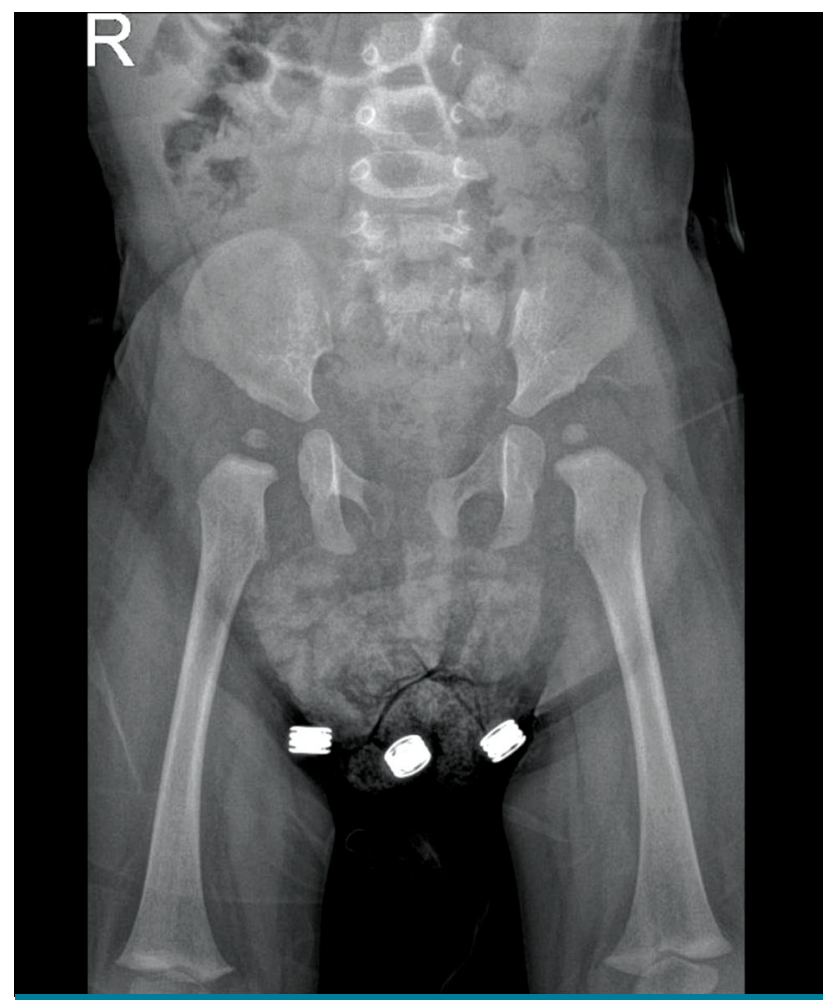

FIGURE 3. A Pelvic radiograph in the ninth month of follow-up showing persistent acetabular dysplasia.

treatment. Demographic and baseline characteristics and treatment results of the patients are presented in Table I.

A four-month-old female infant who was diagnosed with bilateral DDH and underwent radiography in an external center was referred to our hospital to confirm the diagnosis of DDH and for treatment (Patient No. 14). Ultrasonography

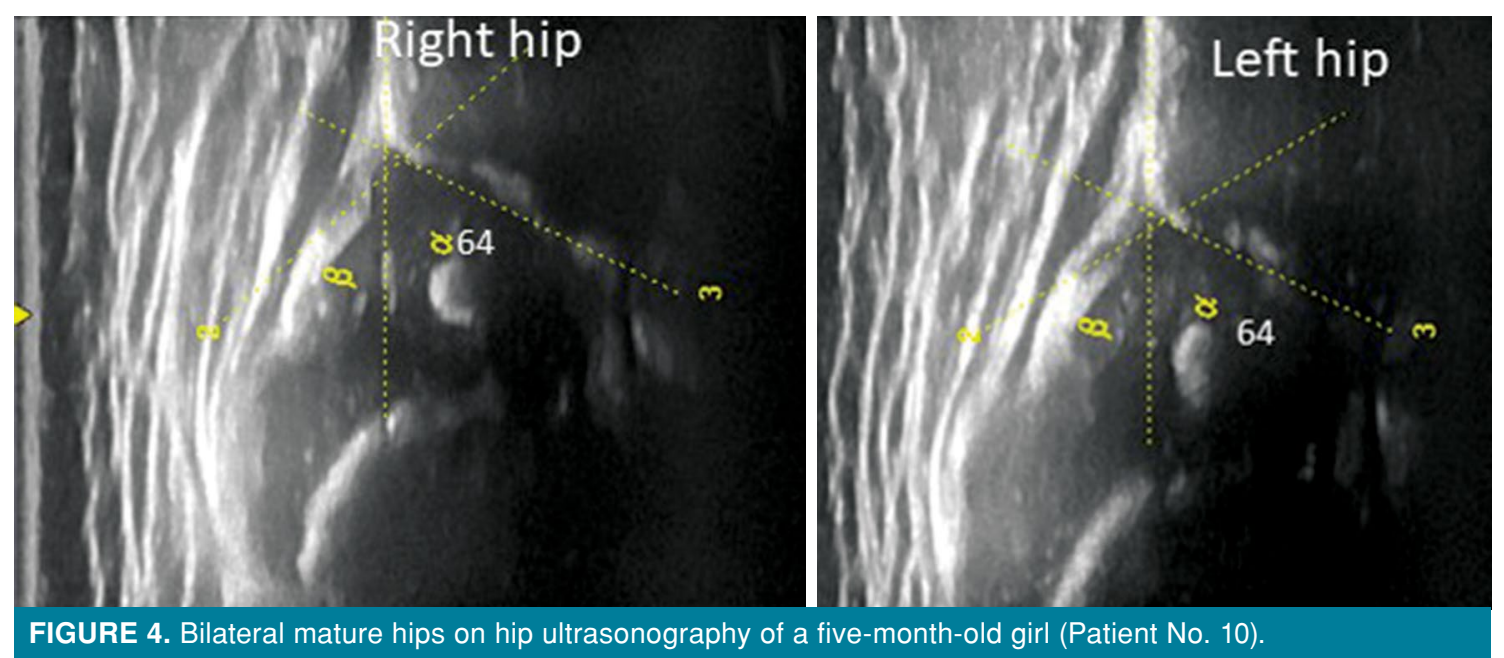



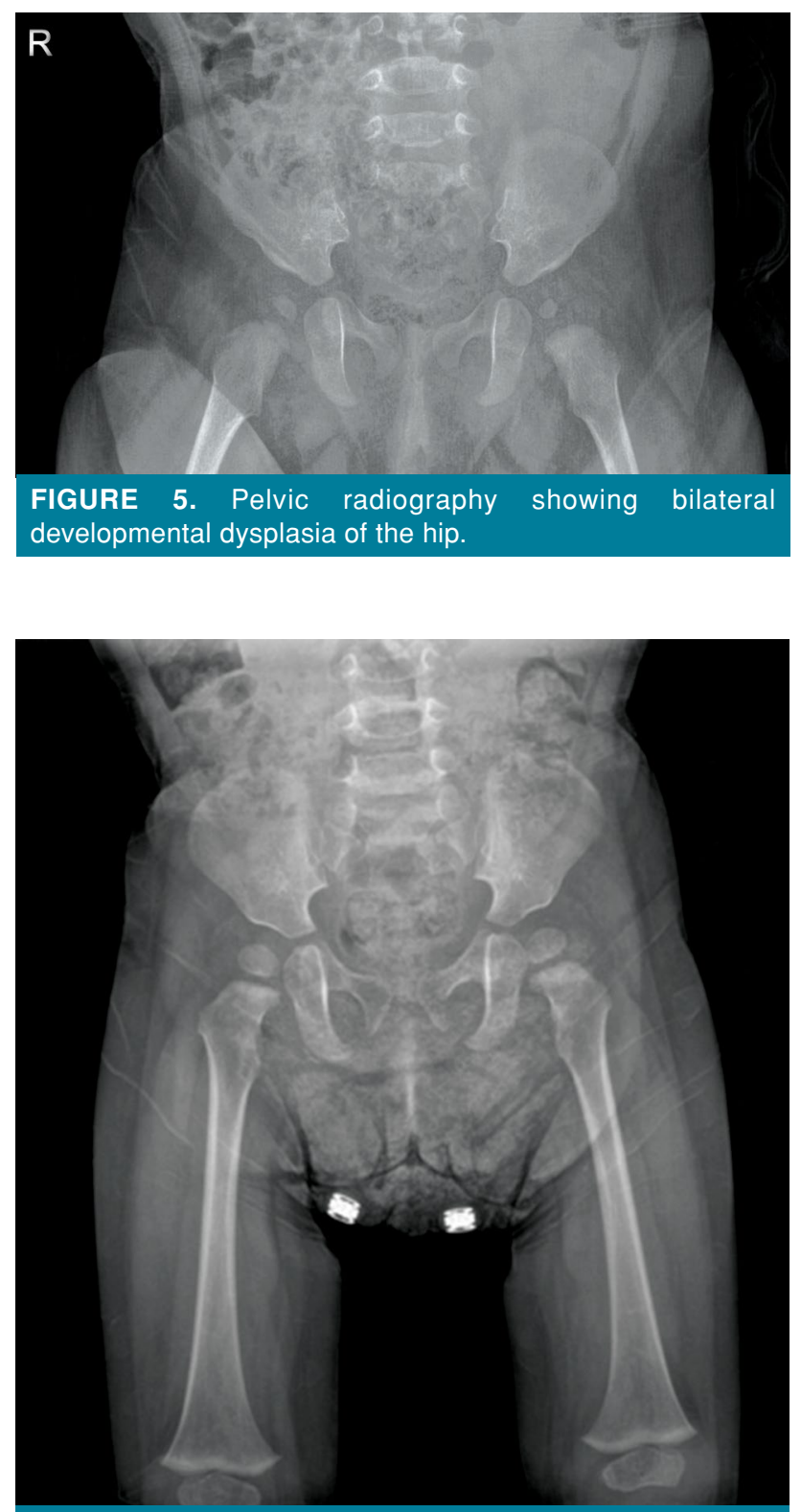

FIGURE 6. Hip development was normal on left hip, but dysplasia of the right hip was seen after 10 months of treatment.

which was performed in our center for the right hip was consistent with the Graf type I and left hip was consistent with Graf type IIb, while simultaneous radiography showed bilateral acetabular dysplasia. Treatment with abduction splint was started based on the diagnosis of radiographic dysplasia. Radiography and hip ultrasonography were performed two months after treatment. Although hip ultrasonography could detect mature type I hip in the right and left side, dysplasia continued in radiography in both hips (Figures 1 and 2). Closed reduction and hip spica

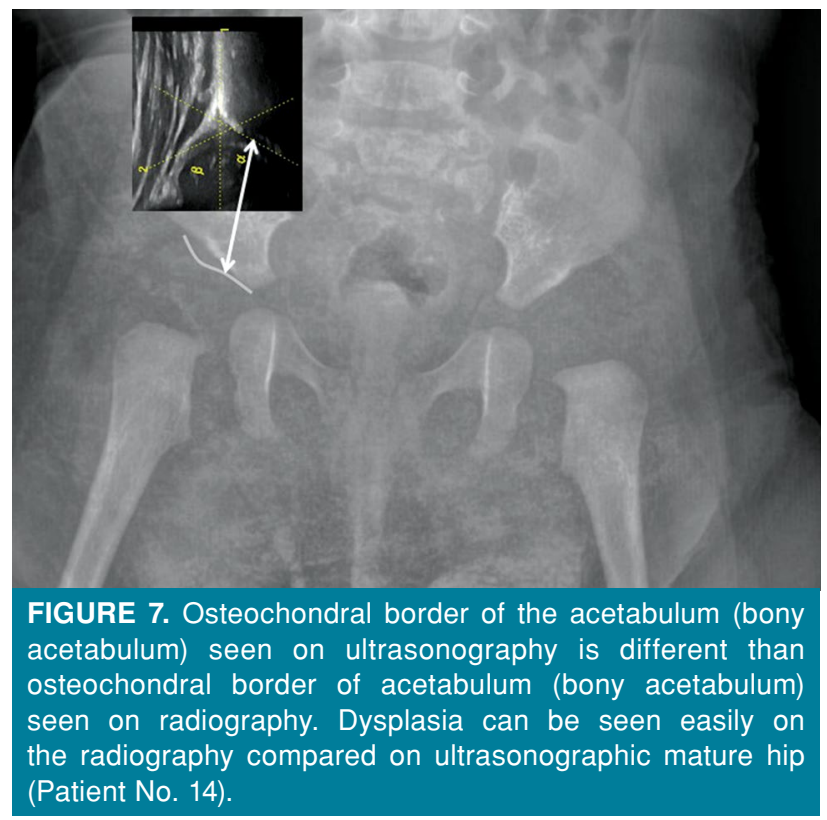

cast were applied for two months. At the end of nine months of follow-up, dysplasia of the both hips persisted (Figure 3).

In addition, a five-month-old girl was referred from pediatric department to our clinic due to the limited abduction in both hips (Patient No. 10). While hip ultrasonography was compatible with maturation in both hips, dysplasia was detected on radiography (Figures 4 and 5). Tübingen hip flexion splint was applied for the treatment and acetabular development was found to be normal in the left hip after 10 months; however, dysplasia was persistent in the right hip (acetabular index 29 degrees in the right hip and 26.5 degrees in the left hip) (Figure 6).

\section{DISCUSSION}

Hip ultrasonography is the most widely used and reliable imaging tool for early diagnosis of DDH. It is a non-invasive and radiation-free tool and can show soft tissue of the joint better than radiography. ${ }^{[1]}$ Thus, many researchers have placed importance on the previous method. Nevertheless, some of the authors have suggested that ultrasonography is too sensitive and shows clinically unimportant instability of the hip joint. ${ }^{[16-18]}$ The ultrasonic beam is aimed at coronal direction to produce section pictures, which would correspond of anteroposterior radiographic views. ${ }^{[19]}$ Ultrasonic waves give an influence of the contours of the bony acetabulum. ${ }^{[20]}$ Acetabular roof, which is sharp and well-defined, represents mature hip joint both on ultrasonography and radiography. 
Ultrasonographic ossification is detected earlier than radiographic ossification. ${ }^{[3]}$ Hip ultrasonography can show the cartilaginous calcification of the tissues more sensitively and differently than the hip radiography. Therefore, according to the Dr. Graf, ${ }^{[3]}$ ultrasound and radiography images which are taken on the same day may be deceptive for an accurate comparison. Figure 7 depicts the hip joint seen on radiography and ultrasonography. According to this figure, osseous acetabulum borders seen on both projections are different. Alpha angle measured by ultrasonography takes into account the osseous acetabulum which is seen on ultrasonography; therefore, simultaneous radiography may show dysplasia, even if hip ultrasonography turns out to be consistent with ultrasonographic hip maturation. Examination of the radiography and ultrasonography shows that the region of calcification which ultrasonography is different than that is shown by radiography and, thus, a hip diagnosed with dysplasia by radiography may be found mature according to the Graf method.

Both the acetabulum development described by Tönnis ${ }^{[15]}$ and the acetabulum development described by Dr. Graf ${ }^{[3]}$ take into account the appearance of the osteochondral acetabulum borders in the coronal hip joint sections. However, there is a discrepancy between the two imaging methods due to the different boundaries of the acetabular calcification seen on coronal images. To the best of our knowledge, there is no study in the literature to evaluate acetabular indices and alpha angles of the same infants by months. Tönnis ${ }^{[15]}$ defined normal and pathological acetabular indices for each hip (left or right) of children (boy or girl) for different ages. However, in the Graf method, only a single angle (alpha) is used to definition of mature hip. Thus, an angle above 60 degrees indicates good acetabular calcification for both sexes and hips.

Review of the literature reveals articles reporting late-diagnosed DDH following normal ultrasonographic hip maturation. Gwynne Jones et al. ${ }^{[8]}$ reported five infants in whom ultrasonographic examination showed a mature hip. On their radiographic examination, at the age of four to six months, DDH was detected. In a recent study, Morris et al. $^{[13]}$ reported that late DDH occurred after normal ultrasonographic hip maturation in infants with breech presentation. Imrie et al. ${ }^{[12]}$ also reported 131 patients with a breech presentation. They had all normal ultrasonographic findings at the initial evaluation. On their control radiography at the age of four to six months, they found $29 \%$ hip dysplasia requiring Pavlik harness treatment. The authors reported that, in infants with risk factors, dysplasia could be detected in radiological follow-up, even if normal hip development was seen on the first hip ultrasonography. However, some authors recommended the cease of follow-up of infants, despite a family history of DDH, when a normal ultrasonographic maturation was obtained in the initial examination. ${ }^{[21,22]}$ In the neonatal period, there are less physiological loads on hip joints in superolateral direction and the remodeling capacity of the hip joint is higher. Therefore, if a hip is seen mature on ultrasonography, its development would form normally over time. ${ }^{[14]}$ However, as the child grows, the acetabular remodeling capacity decreases and the physiological load increases on the hip joint. According to Dr. Graf, ${ }^{[3]}$ hip development is better visible on the ultrasound taken on the same day as the X-ray. Our findings also confirm this concept. However, normal acetabular development seen on ultrasonography did not guarantee that acetabulum calcification would be normal in infants above four months of age who were at risk for DDH. We also support the previously reported hypothesis ${ }^{[23]}$ that "the only guarantee of a lifetime of normal hip function is a completely normal radiographic appearance of the hip". ${ }^{[24]}$ It is also possible that some of our patients treated according to the radiography would have had normal acetabular development in a longterm follow up. However, it is noteworthy that we included infants whose hip dysplasia persisted, despite treatment. We believe that anteroposterior radiography of the pelvis would be a better approach to confirm mature hips in infants at risk for DDH and at an age where bony details can be seen.

In conclusion, DDH may continue in infants who are at risk and older than four months of age, even if there is ultrasonographic maturation and, therefore, some cases may need to be treated. For a more comprehensive evaluation, further large-scales, prospective studies are needed.

\section{Declaration of conflicting interests}

The authors declared no conflicts of interest with respect to the authorship and/or publication of this article.

\section{Funding}

The authors received no financial support for the research and/or authorship of this article.

\section{REFERENCES}

1. Graf R, Mohajer M, Plattner F. Hip sonography update. Quality-management, catastrophes - tips and tricks. Med Ultrason 2013;15:299-303. 
2. Kaymaz B, Bakırcığlu S, Yılmaz G, Atay ÖA, Aksoy MC. Osteochondral autograft transplantation of the femoral head in sequelae of developmental dysplasia of hip: A case report and review of the literature. Jt Dis Relat Surg 2020;31:619-25.

3. Graf R. Mesurement technique. In: Graf R, editor. Hip sonography diagnosis and management of infant hip dysplasia. 2nd ed. Berlin: Heidelberg, Springer-Verlag; 2006. p. 46-50.

4. Wientroub S, Grill F. Ultrasonography in developmental dysplasia of the hip. J Bone Joint Surg Am 2000;82-A:1004-18.

5. von Kries R, Ihme N, Oberle D, Lorani A, Stark R, Altenhofen $\mathrm{L}$, et al. Effect of ultrasound screening on the rate of first operative procedures for developmental hip dysplasia in Germany. Lancet 2003;362:1883-7.

6. Thallinger C, Pospischill R, Ganger R, Radler C, Krall $\mathrm{C}$, Grill F. Long-term results of a nationwide general ultrasound screening system for developmental disorders of the hip: the Austrian hip screening program. J Child Orthop 2014;8:3-10.

7. Wenger D, Düppe H, Nilsson JÅ, Tiderius CJ. Incidence of late-diagnosed hip dislocation after universal clinical screening in Sweden. JAMA Netw Open 2019;2:e1914779.

8. Gwynne Jones DP, Dunbar JD, Theis JC. Late presenting dislocation of sonographically stable hips. J Pediatr Orthop B 2006;15:257-61.

9. Rafique A, Set P, Berman L. Late presentation of developmental dysplasia of the hip following normal ultrasound examination. Clin Radiol 2007;62:181-4.

10. Jaiswal A, Starks I, Kiely NT. Late dislocation of the hip following normal neonatal clinical and ultrasound examination. J Bone Joint Surg [Br] 2010;92:1449-51.

11. Brinsmead T, Hong T, Frawley K. A case of late presentation of developmental dysplasia of the hip with normal screening ultrasound scan. J Paediatr Child Health 2014;50:494.

12. Imrie M, Scott V, Stearns $P$, Bastrom $T$, Mubarak SJ. Is ultrasound screening for $\mathrm{DDH}$ in babies born breech sufficient? J Child Orthop 2010;4:3-8.

13. Morris AR, Thomas JMC, Reading IC, Clarke NMP. Does late hip dysplasia occur after normal ultrasound screening in breech babies? J Pediatr Orthop 2019;39:187-92.

14. Atalar H, Gunay C, Turanli S, Koktener A. Discrepancy between ultrasonographic and radiographic findings in patients treated for developmental dysplasia of the hip; hip maturation on ultrasonography may not be consistent with radiographic hip maturation. J Pediatr Orthop B 2020;29:228-34.

15. Tönnis D. Normal values of the hip joint for the evaluation of X-rays in children and adults. Clin Orthop Relat Res 1976;(119):39-47.

16. Andersson JE, Funnemark PO. Neonatal hip instability: Screening with anterior-dynamic ultrasound method. J Pediatr Orthop 1995;15:322-4.

17. Berman L, Klenerman L. Ultrasound screening for hip abnormalities: Preliminary findings in 1001 neonates. Br Med J (Clin Res Ed) 1986;293:719-22.

18. Clarke NM, Clegg J, Al-Chalabi AN. Ultrasound screening of hips at risk for $\mathrm{CDH}$. Failure to reduce the incidence of late cases. J Bone Joint Surg [Br] 1989;71:9-12.

19. Graf R. Classification of hip joint dysplasia by means of sonography. Arch Orthop Trauma Surg 1984;102:248-55.

20. Graf R. The diagnosis of congenital hip-joint dislocation by the ultrasonic Combound treatment. Arch Orthop Trauma Surg 1980;97:117-33.

21. Arumilli BR, Koneru P, Garg NK, Davies R, Saville $\mathrm{S}$, Sampath J, et al. Is secondary radiological followup of infants with a family history of developmental dysplasia of the hip necessary? J Bone Joint Surg [Br] 2006;88:1224-7.

22. Osarumwense D, Popple D, Kershaw IF, Kershaw CJ, Furlong AJ. What follow-up is required for children with a family history of developmental dysplasia of the hip? J Pediatr Orthop B 2007;16:399-402.

23. Herring JA. Developmental dysplasia of the hip. In: Herring JA, editor. Tachdjian's pediatric orthopaedics. 4th ed. Philadelphia: Saunders Elsevier; 2008. p. 649-51.

24. Atalar H, Günay C, Atik OŞ. Is treatment termination safe in developmental dysplasia of the hip following ultrasonographic normalization? Jt Dis Relat Surg 2021;32:521-2. 